\title{
Controller of Register Combinations and Tone Keys for Pipe Organ
}

\author{
Ernest Gungl, Zmago Brezočnik \\ Faculty of Electrical Engineering and Computer Science \\ University of Maribor \\ Maribor, Slovenia
}
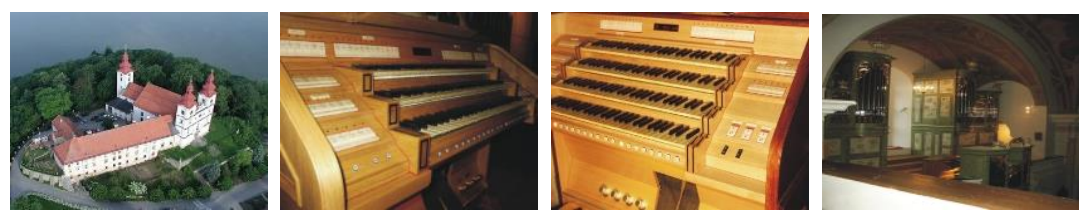

\begin{abstract}
A pipe organ is a musical instrument that produces sound by driving pressurized air through the organ pipes selected from a keyboard called a manual. It is constructed from settled groups of pipes. Each group is composed of similar pipes with the same tone colour and loudness but different pitch. Such a group is called a rank. We have developed two electronic devices for upgrading the organ. The first device named Controller of Register Combinations is intended for storing rank combinations and pipe organ controlling. The second device named Controller of Tone Keys for pipe organ allows users to play the organ simultaneously on two separate keyboards. In this paper, we represent the purpose, scheme, and our realization of both devices. The correct functioning of the devices was proved by integrating them into a church organ. We have already equipped several church organs with our electronics, and they all work flawlessly. Feedback from the organists is excellent, as both Controller of Register Combination and Controller of Tone Keys make it easier for them to play. The success so far and the positive responses of the organists have encouraged us already to plan further improvements and upgrades of the organ electronics.
\end{abstract}

Keywords:- Pipe Organ, Pipe Organ Electronics, Controller of Rank Combinations, Controller of Tone Keys.

\section{INTRODUCTION}

The first pipe organ was created 246 years BC [1]. It was made by Ctesibius of Alexandria in Asia Minor, who was not a musician but a technician. He used water trapped under the bell to regulate air pressure what was replaced by blacksmith's bellows in the 1st century AD [1] when also the possibility of choosing different ranks was added with Vitruvius' organ. With the invention of the dividing board in the 14th century, the width of the manual coincided with today's manual. Previously, the tone key was placed in front of the flute. Around 1300, a pedal manual for playing long bass notes on deep burgundy flutes was discovered [1]. In the 19th century, organ blinds were also born, which close the flutes in the cupboard and allow us to change the volume of the flute captured behind the blind by opening and closing them with the foot. At that time, a crescendo was created, where we also use the foot to activate and deactivate individual ranks (from the quietest to the loudest). The invention of the mechanical tracker-a mechanism for transferring an organist's actions to the ranks and pipes valves, allows precise control over the pipe speech [2]. Pneumatic trackers and electro-pneumatic trackers in the 19th century allowed the console to be moved away from the flutes, allowing new possibilities for a pipe organ placement. However, pneumatic trackers also introduced delays to the organist actions, so organ builders returned to mechanical trackers used in Baroque and to electrical trackers in the 20th century $[1,2]$.

Although some new pipe organs are still made as they were centuries ago, many of them have been upgraded with modern electronics and other inventions. MIDI-enabled pipe organ console with software support for a gesture recognizable interface is proposed in [3]. In [4], a pipe organ sound captured by a microphone, preprocessed in Digital Signal Processor, and played back in a speaker is combined with an original pipe organ sound to achieve a unique sound effect. Imitation of a complete set of organ ranks using additive synthesis is proposed in [12]. Some producers of pipe organ devices $[5,6,7]$ also sell or produce electronic control systems for pipe organ small to large, including those with multiple consoles. The solution to the problem of musical articulation limitations in a pipe instrument with electromagnetic actions is proposed in [14].

In close collaboration with organists, persons who play the pipe organ musical instrument, we have developed two such modern devices for pipe organ, called the Controller of Rank Combinations (CRC) and the Controller of Tone Keys (CTK). CRC simplifies the organist's work in playing complex compositions. Complete control of a pipe organ with a large number of ranks is almost impossible without such a device. CTC allows two or more organists to simultaneously play the pipe organ from different locations - in our case, with a distance of about $25 \mathrm{~m}$ between the organists. 
In order to better understand the purpose and operation of the CRC and CTK, we first explain the organ terminology [11] and the principle of operation of the pipe organ using a scheme in Fig. 1. The blower, driven by a quiet electric motor, fills the reservoir (bellows), which serves as compressed air storage. From here, a compressed air (wind) travels through trunks and enters a windchest, a mechanism used for admitting wind to selected pipes.

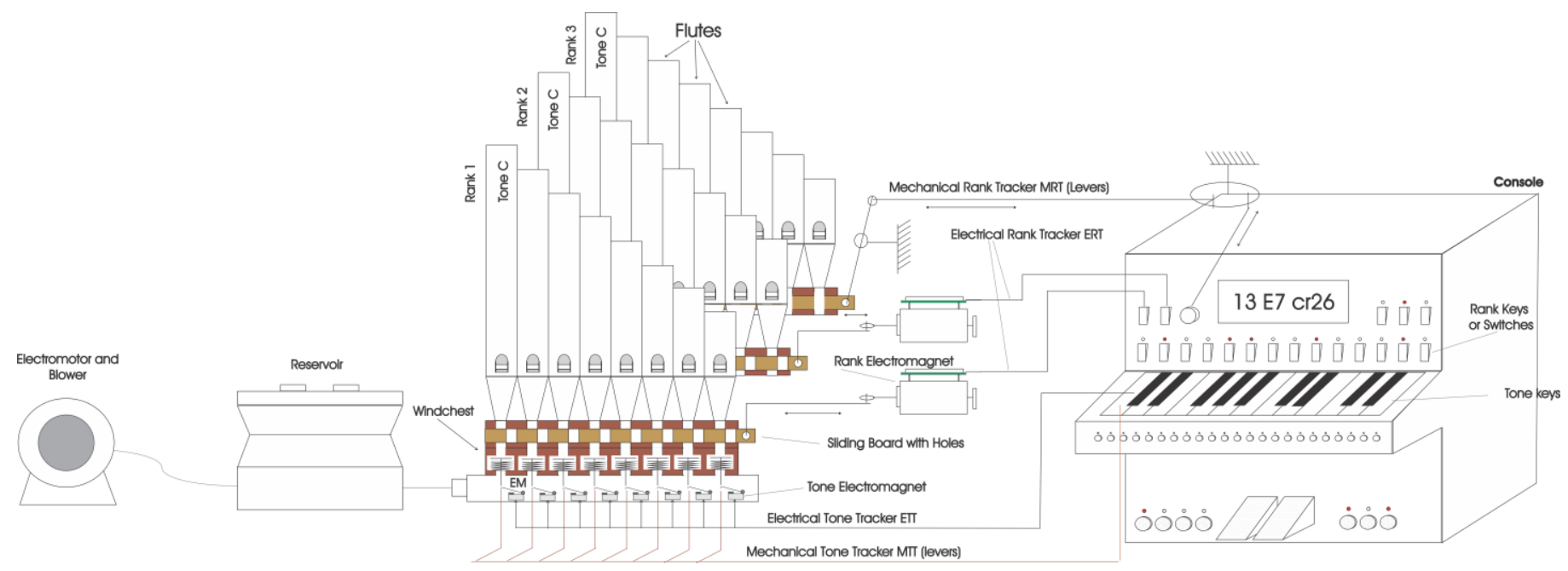

Fig. 1: A principle of operation of the pipe organ

By pressing a black or a white tone key on a manual of a console, an associated electromagnet opens a valve, and the wind continues into a ventricle common to all eponymous tones of all ranks. The tone is played only by those flutes whose ranks are activated by movable sliders. Different ranks imitate different musical instruments, like a trumpet, trombone, etc. Slider activates and deactivates a rank by opening and closing holes to the pipes above it. Because the windchest part under the slider is common to all the ranks, it is drawn just under the first rank in Fig. 1. In general, an opening/closing of the slider or the tuning valve is achieved by mechanical and/or electrical tracker. A tracker is a mechanism for transferring actions from an organist (a console) to the windchest. It is also known as a key action when a tone key is pressed, or a stop action, when a rank key is pressed [2]. Since the action can be mechanical, electrical, pneumatical, optical, or composed of any combination of them, we name the trackers by the type of action. When an organist presses a rank key and a black/white tune key, a tracker opens a register and a valve of a tone ventricle, respectively. As shown in Fig. 1, a mechanical (levers) and electrical trackers (electromagnets) are used.

An essential element of a pipe organ is the flute [8]. The flute generates a fundamental frequency tone and also higher harmonic ones [9]. Equation (1) describes fundamental relations between speed $v$, the frequency $f$, and the wavelength $\lambda$ of a generated sound wave. A fundamental frequency and higher harmonic frequencies for open (flute) and closed (clarinet) pipes are described by (2) and (3). $L$ represents the physical length of the flute, where $n$ is a nonnegative integer.

$$
v=\lambda \cdot f \Rightarrow f=\frac{v}{\lambda}
$$

$$
\begin{aligned}
& f_{n}=(n+1) \frac{v}{2 L} \\
& f_{n}=(2 n+1) \frac{v}{4 L}
\end{aligned}
$$

The organist operates the organ system via a console consisting of a cabinet containing one or more manuals, rank keys (stops), function keys, pedal keyboards, a crescendo, a blind window pedal, and a display for showing the current status of the instrument. The process of setting different combinations of the ranks by using rank keys is known as organ registration. Function keys serve for storing, recalling, and manipulating rank combinations and other things, like user login, MIDI activation, etc. The blind window pedal allows an organist to change the volume of the flute located behind the blind window by opening and closing it.

Crescendo is a special type of pedal sequencer. By moving the pedal, we switch between 30 programmable rank combinations. Unlike the classic sequencer, which allows stepping between rank combinations stored in consecutive memory entries, which are not necessarily related, the rank combinations for crescendo entries are well related. An adequately programmed crescendo allows an organist to switch between the louder and quieter part of the song by just moving the pedal.

All the components of a console are connected to CRC and CTK. Their functional description and structure are described in the next chapter. Findings regarding the realization of the initial set goals after installing devices into the actual pipe organ are given in Chapter II. In Chapter III, the paper concludes by stating the last upgrades of devices and our plans for future improvements. 


\section{THE PROJECT OUTLINE AND IMPLEMENTATION}

A. The Physical Layout of the Project

We started our research by collecting requirements about the final pipe organ project. Unfortunately, the requirements have been upgraded over time and have become increasingly complex. Fig. 2 shows the final requirements of a project we had to realize.

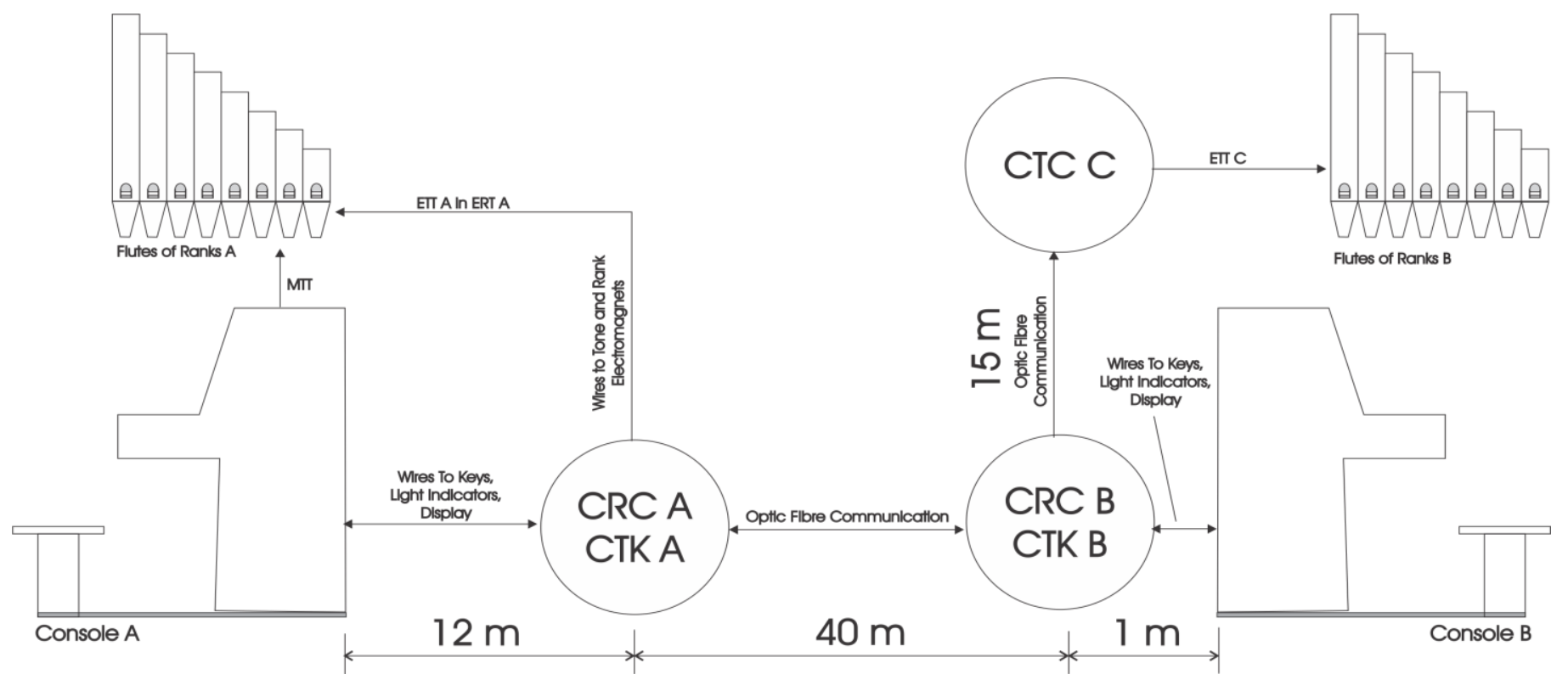

Fig. 2: The final requirements of a project in terms of the physical layout

The project contains two consoles $25 \mathrm{~m}$ apart (air distance) and two dislocated sets of register ranks. An optical connection is used for communication between dislocated units via CRC and CTK devices. CRC A and CTK A are logically connected and form a master device, while all other communication devices are slaves. Master combines actions from both consoles and produces a required result that is forwarded to both for their status indication and for activating pipe ranks. All commands generated by console $\mathrm{B}$ are forwarded to CRC/CTK A. CRC $\mathrm{B}$ is physically identical to CRC $\mathrm{A}$ and is used as an interface for console B. Console A and console B are fully transparent for both organists. CRC and CTK, among others, represent an optical tracker. Console A uses an electrical and mechanical rank tracker and only an electrical manual tracker for tone keys. Electrical trackers, in combination with optical ones, are needed for dislocated consoles.

\section{B. CTC and CRC registration}

CTK is a more straightforward device. It collects states of all manuals' black and white keys and pedal keyboards for both consoles and forwards this information through other CTKs to the pipes. It also communicates with CRC for MIDI information exchange. Pipes' tone magnets are connected to CTC through the electrical tracker. CRC connects all other elements of a console (Fig. 1). In the process of programming a rank registration, an organist selects the desired combination of ranks using rank keys and stores it using function keys in memory under the label name, for example, E7. While playing an instrument, programmed rank combinations can be recalled. We provide different schemes for labelling a memory location. A similar process is used for crescendo programming, but instead of function keys, a pedal is used which is connected to function inputs of CRC. There are two crescendos, A and B. The first one forces a new rank combination for each pedal position, but the second one just activates new ranks without turning off initial ones. Blinder controller is not programmable and is also connected to CRC. An import part of a console is a display which is connected to CRC using RS232 physical layer and provides an organist with all the status information of the instrument. Among others, CRC contains MIDI interface for information exchange with PC, memory card reader for storing individual organist's registration information to memory cards and ethernet interface for Internet connectivity.

The principle of nesting certain rank functions for two examples (red and blue lines) is shown in Fig. 3. After turning on an organ instrument, we can manually set some rank combination by using rank buttons. Activating button for crescendo A takes us to state $\mathrm{Cr} \mathrm{A}$. From here we can return to the initial state or continue to a different state. So, by activating/deactivate a particular function, we enter/leave a particular state. Some states can only be visited from certain states. For example, we can enter the state "Add Reed Pipes" or "Remove Reed Pipes" only from state "Tutti". In the diagram, tutti function activates most of the available ranks, "Pleno" is suitable for playing Baroque compositions, but the function "Add Reed pipes" (not shown in Fig. 3) or "Remove Reed pipes adds or removes ranks of pipes that comprise a metal tongue (the reed). An important 
function is a sequencer which allows an organist to step through a sequence of memory locations.

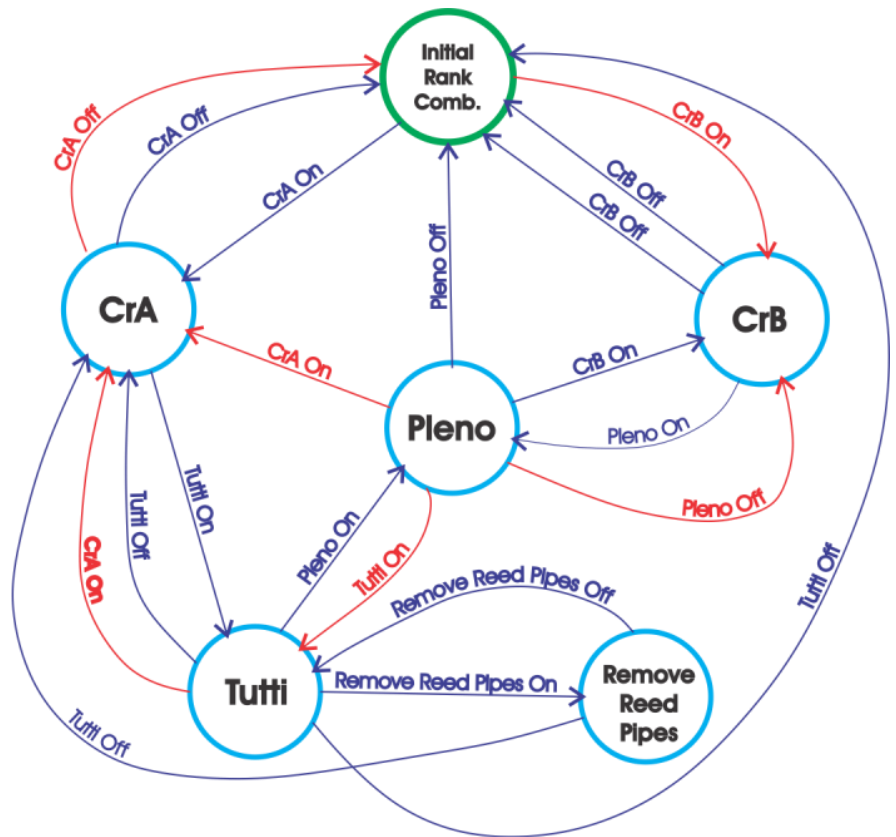

Fig. 3: Nesting procedure for certain rank functions
C. Implementation and Inclusion of CRC/CTK in a Project

There are two cards of CRC (Fig. 4) and seven cards of CTK (Fig. 5) embedded in the project. Each CRC card has 112 outputs and 112 inputs. Each input and output CTK card also has 112 input or output lines, respectively. All input and output lines use optocoupler devices to improve robustness. Eight bits are read (input card) or write (output card) at the same time using a standard 8-bit bus connected to a microcontroller. Eight bits are selected using a line decoder (LD) controlled by a microcontroller. For input cards emitters of 8 input optocouplers are connected to one output line of LD and pulled up collectors are connected to an 8-bit data bus. When LD selects a particular octet, eight optocouplers are in a working condition so that the microcontroller can read the octet on the bus. For output cards microcontroller put 8-bit data to a bus and use LD to select a particular octal D-type latch to move data on the bus into a latch. All latches' enable pins are pulled down to prevent uncontrolled switching of their outputs and indirectly moving of rank electromagnets at startup of an electronic system.

Some function keys can be used by feet. Although we talk only about simple rank keys, there were also used different variants of them, like mechanical imitation switches, etc. Some of them require special treatment from an electronic and a programming point of view.

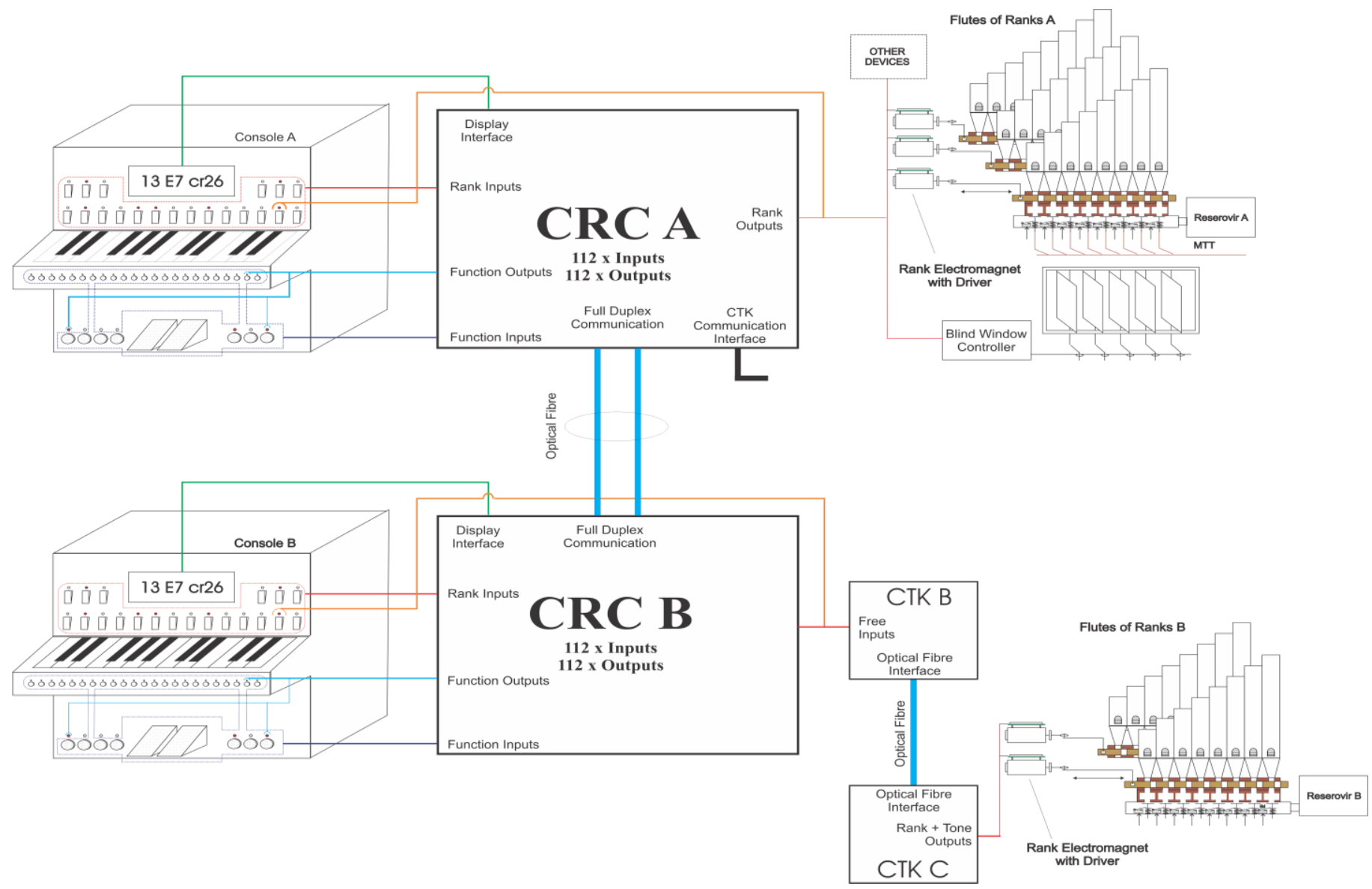

Fig. 4: CRCs connection to Consoles and their communication 
Fig. 4 shows blind window controller and CRC B that borrows CTK to transfer its information (together with primary CTK information) to dislocated pipes B. Console A has three and console B four manuals (Fig. 5) which are connected to input CTK cards. Drivers of output cards drive tone electromagnets. Arrows show communication direction between cards. In addition to an electrical tone tracker, the mechanical one is used for console A.

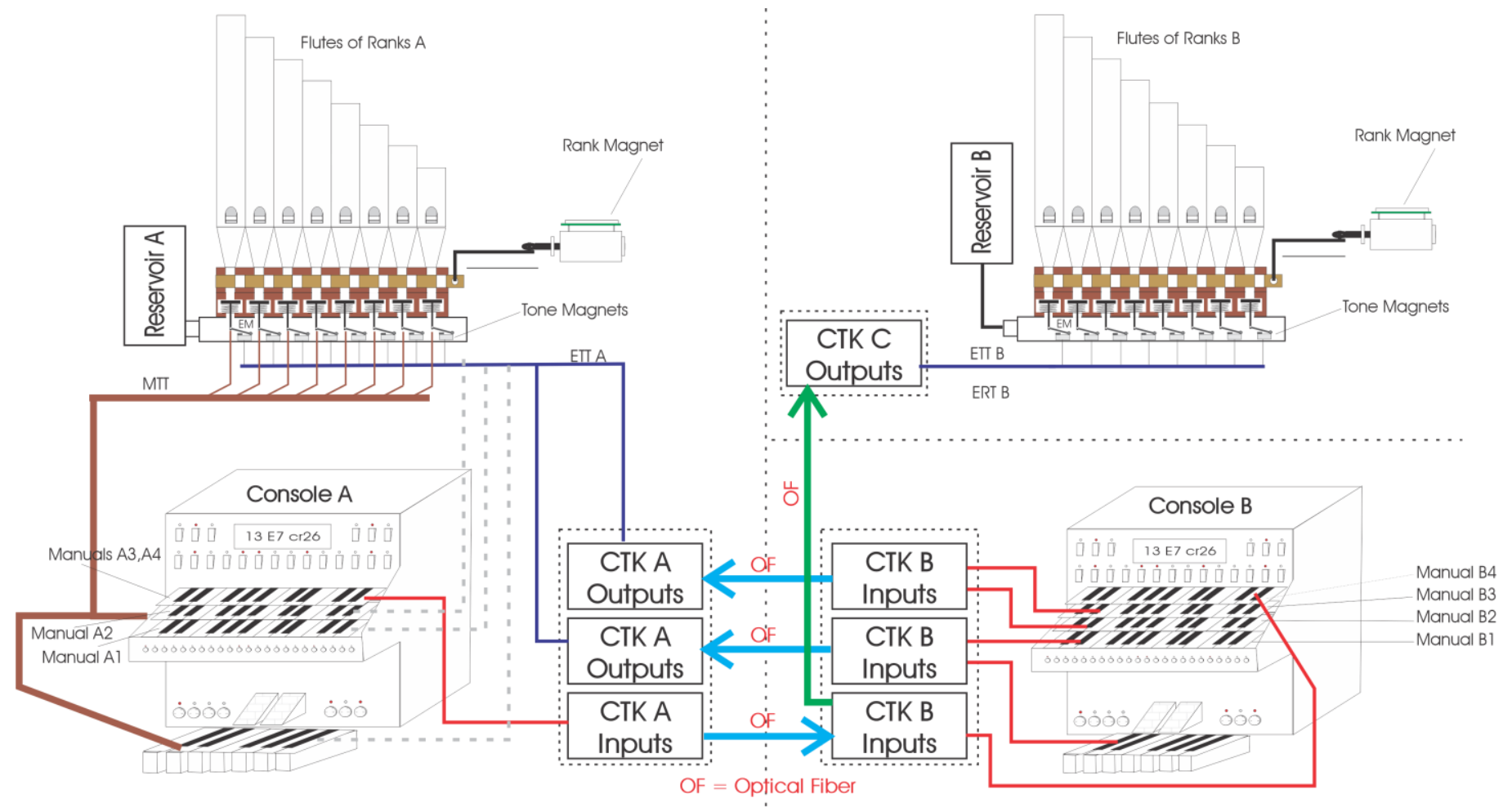

Fig. 5: CTKs connection to Consoles and their communication

The communication protocol is based on bit stuffing [10], which ensures that the bit sequence intended to indicate the beginning of the data block is uniquely recognizable within the bitstream between the sender and the receiver. The 8-bit value 10000001 (initial flag) is used to mark the beginning of the data block. Cyclic Redundancy Check is used for error detection of a received data block. If a received block contains an error, it is simply discarded without informing a sender about a communication problem, and a new block is awaited.

\section{Results and Improvements}

After the realization of the devices and installing them into the new pipe organ, we started testing the operation of the entire communication system. Although the electronic devices were tested in parts before being installed in a real pipe organ, reliable testing was not feasible due to the overall complexity of the system. Initial testing showed that some of the requirements were misunderstood because we did not know the organ well enough in the beginning.

Part of the whole embedded electronic system built years ago when a new organ was constructed is shown in Fig. 6, while most of the rank and function keys are shown in Fig. 7.

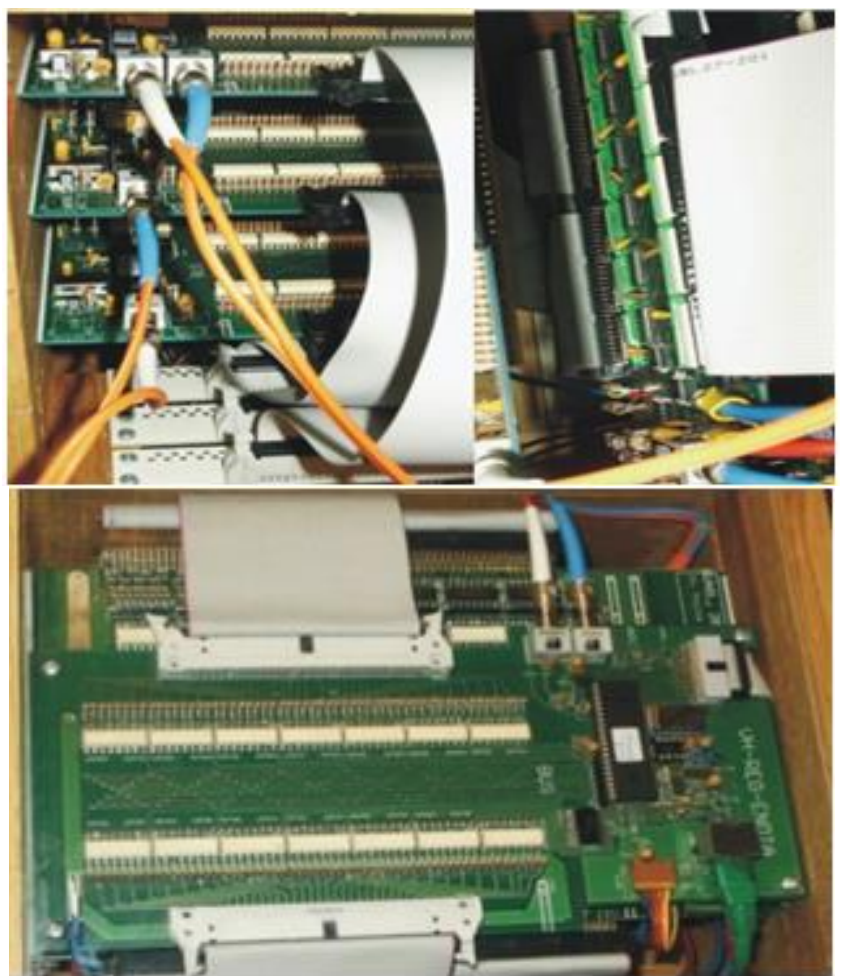

Fig. 6: CRC, input and output CTK cards with optical connections 
ISSN No:-2456-2165
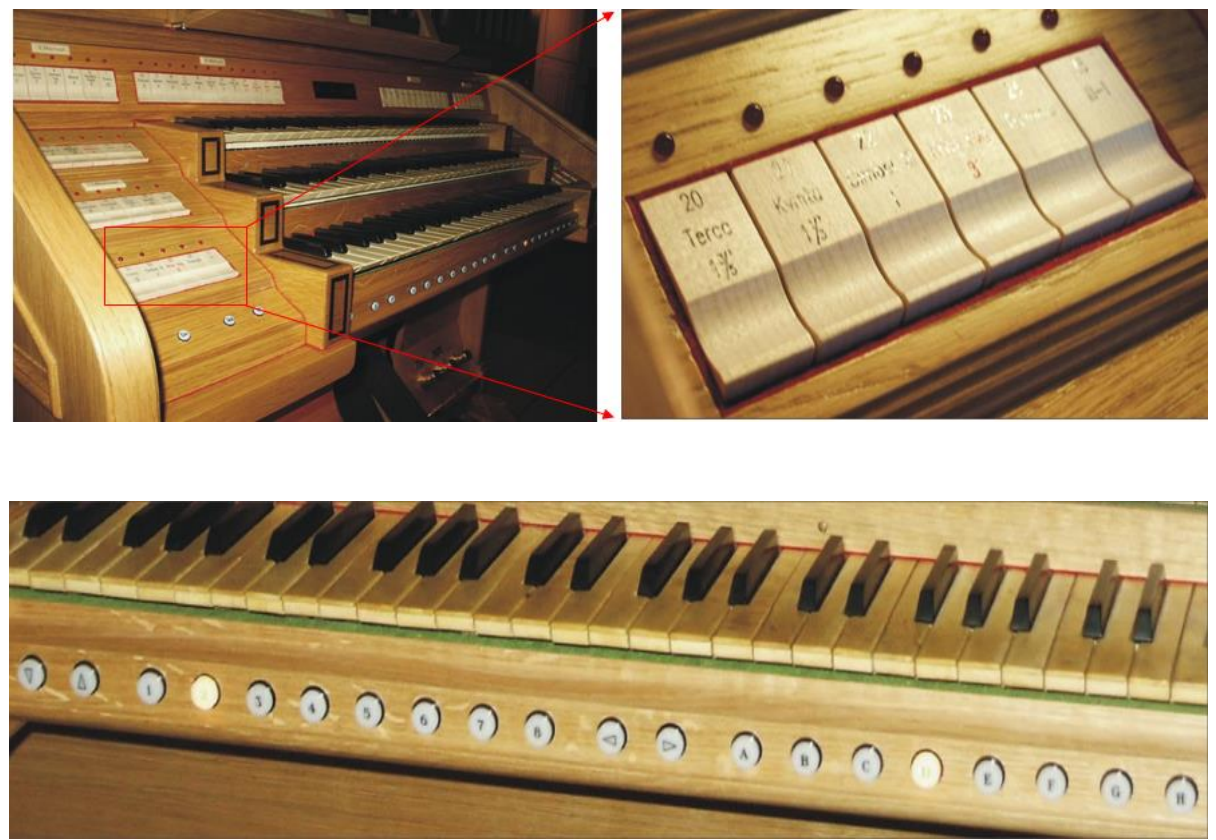

Fig 7: Rank and function keys on a real organ

\section{CONCLUSION}

Although the project was quite complex-there were 54 ranks, 3271 flutes, two consoles with three and four manuals, 1232 input/output lines of CRC/CTK, 13,000 solder joints and many hours of work, it was worth participating.

It has been 17 years since the first installation of the organ with our electronics in the church. Many hours of playing and concerts of various ranks were performed on them, even in a duet, without any problems. We have learned a lot about organ technology, which helps us to develop new ideas. Organ electronic system received many upgrades, and one of our last designs is shown in Fig. 8.

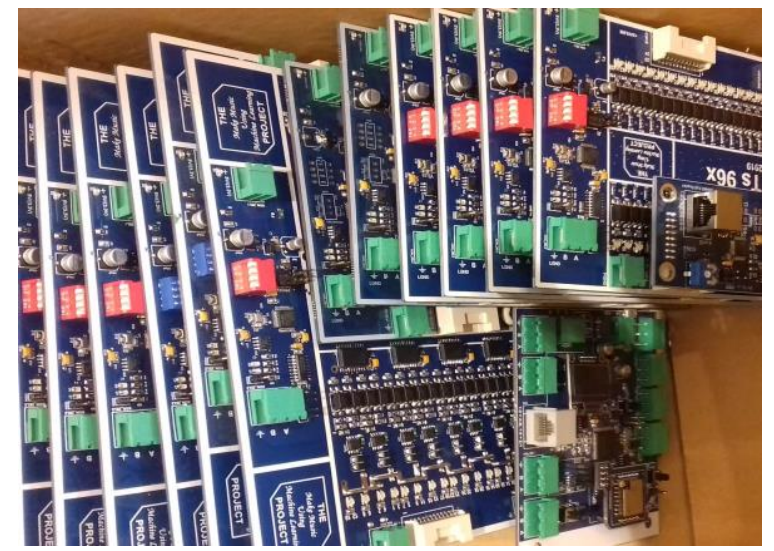

Fig. 8: Upgraded organ electronic cards

The optical interface is replaced by a cheaper Ethernet one. There is no difference between rank and tone cards which are connected to the master card. Master cards can communicate wirelessly using 802.11 standard. Cards are connected to the master card using RS485 physical interface, which allows that many cards can be connected to it.
Moreover, song recording on SD card and replay have been integrated into a master card.

The electronic system supports everything needed to implement Machine learning techniques, like Long ShortTerm Memory type of Recurrent Neural Networks, that can be learnt to generate pipe organ music itself [13] or using a Reinforcement Learning to help organists in doing rank registration which is our current project plan.

\section{REFERENCES}

[1]. E. Škulj, "Orglarstvo" (in Slovene), Družina, Ljubljana, 1992

[2]. "Pipe organ," https://en.wikipedia.org/wiki/Pipe_organ\#History_and development, 30. 7. 2020.

[3]. J. Wang, "Explorations in digital control of MIDIenabled pipe organs," Proceedings of the 14th Sound and Music Computing Conference, July 5-8, Espoo, Finland.

[4]. A. Blackburn, "Computer use in music for the pipe organ and real time dsp - or the music of Janus," Proceedings of the CreateWorld 2009 "Mobile Me Creativity on the Go", 30 November - 2 December, Brisbane, Australia.

[5]. "Classic organ works," https://www.organworks.com/, 30. 7. 2020

[6]. "Orgelteile Heuss," https://www.ottoheuss.de/, 30. 7. 2020.

[7]. "Pipe organ parts," https://laukhuff.de/, 30. 7. 2020.

[8]. J. Angster, "Acoustics of organ pipes and future trends in the research," Fraunhofer-Institut für Bauphysik (Fraunhofer Institute for Building Physics IBP), Nobelstrasse 12, 70569 Stuttgart, Germany.

[9]. "Open vs Closed pipes (Flutes vs Clarinets)," https://newt.phys.unsw.edu.au/jw/flutes.v.clarinets.htm 1, 30. 7. 2020.

[10]. P. Branch. S. 7ander. "Bit-stuffing rate in the hiohlevel data link control (HDLC) nrotocol." Centre for Advanced Internet Architectures. Technical Renort 081121A. Swinburne University of Technology Melbourne, Australia. 

[11]. "Glossary of or organ terminology,"

[12]. -building-principles-2019/glossary-of-organ-terms2/\#tab-1, 30. 7. 2020.

[13]. P. Comerford, "Simulating an organ with additive synthesis," Computer Music Journal, Vol. 17, No. 2, 1993, pp. 55-65.

[14]. C. A. Huang et al., "Music Transformer: Generating, Music with Long-Term Structure," arXiv:1809.04281v3 [cs.LG], 12 December 2018.

[15]. B. Kostek, "Intelligent control system implementation to the pipe organ instrument," Proceedings of the International Workshop on Rough Sets and Knowledge Discovery (RSKD'93), Banff, Alberta, Canada, 12-15 October 1993. 